\title{
Kinetic Monte Carlo Study of Hole Transport in Pentacene and its Derivatives
}

\author{
Denis da Mata Oliveira ${ }^{1 *}$ (PG), Luiz Antônio Ribeiro Júnior² (PQ) \\ ${ }^{1}$ Institute of Physics, University of Brasília, Brasília, 70910-970, DF, Brazil and ${ }^{2}$ International Center for \\ Condensed Matter Physics, University of Brasília, P.O. Box 04531, 70.919-970, Brasília, DF, Brazil \\ ${ }^{1}$ denisdamata@yahoo.com.br \\ Keywords: Organic Semiconductors, Pentacene Derivatives, Monte Carlo, Charge Transport.
}

\section{Introduction}

The need for a deeper understanding of the internal processes in organic photovoltaic devices, such as the transport of charge carriers, has driven the development of microscopic numerical models [1]. Especially simulations based on Kinetic Monte Carlo (KMC) techniques provide a powerful and versatile tool to gain further insight into these mechanisms because they allow to represent the complex morphology of bulk-heterojunction blends and to track the dynamic behavior of single particles [1]. Here, the transport of holes in organic molecular crystals (based in pentacene and its derivatives) is theoretically studied in the framework of a model based on the KMC algorithm. We have used the actively researched pentacene crystal [2] as the hole transport layer.

\section{Methodology}

Within the hopping description, the charge transport can be characterized by a diffusion process, in which the charge carrier hops between adjacent molecules according to the charge transfer rates that apply in the absence of an external electric field [3]. In order to account the charge transfer rates, we have implemented in our KMC code the widely used charge transfer rate from the classical Marcus theory [3]. Our KMC method can model the transport of organic materials with several charge carriers interacting [4] and disorder effect, all this with periodic boundary conditions.

\section{Results}

With KMC simulations, we have calculated the mobility dependence on temperature and electric field for different pentacene-based crystals. The obtained mobilities present values that are closer to the literature values, maintaining the same qualitative functional dependence.

\section{Conclusions}

A realistic KMC model for charge transport in organic materials is obtained here. The implementation of long-range electrostatic forces allows to represent the charge interactions in low-dielectric organic materials and makes the model unique in its features. The good agreement between theory and literature, when it comes to 
the mobility values, indicates that our KMC model is able to reproduce the charge transport mechanisms.

\section{Acknowledgements}

The authors gratefully acknowledge the financial support from the Brazilian Research Councils CNPq and FAPDF. L.A.R.J. gratefully acknowledges the financial support from the Brazilian Research Council FAPDF grant 0193.000942/2015 and 193.001.511/2017. L.A.R.J. also wishes to thank the Brazilian Ministry of Planning, Budget and Management (Grant DIPLA 005/2016).

\section{References}

[1] J. L. Brédas et. al. Acc. Chem. Res. 42, 1691-1699, 2009.

[2] V. Coropceanu et. al. Chem. Rev. 107, 926-952, 2007.

[3] R. Volpi. Modeling Charge Transport for Organic Solar Cells within Marcus Theory. PhD Thesis, Linköping University, Linköping, Sweden 2017.

[4] Markus Krammer. Coulomb Interactions in Kinetic Monte Carlo Simulations

for Charge Transport in Organic Semiconductors. Master's thesis, Graz University of Technology, Graz, Austria 2016. 